\title{
Philosophiques
}

\section{José L. Zalabardo, Representation and Reality in Wittgenstein's Tractatus, Oxford, Oxford University Press, 2015, 263 pages}

\section{Jonathan Gombin}

Volume 45, numéro 2, automne 2018

URI : https://id.erudit.org/iderudit/1055288ar

DOI : https://doi.org/10.7202/1055288ar

Aller au sommaire du numéro

Éditeur(s)

Société de philosophie du Québec

ISSN

0316-2923 (imprimé)

1492-1391 (numérique)

Découvrir la revue

Citer ce compte rendu

Gombin, J. (2018). Compte rendu de [José L. Zalabardo, Representation and Reality in Wittgenstein's Tractatus, Oxford, Oxford University Press, 2015, 263 pages]. Philosophiques, 45(2), 569-574. https://doi.org/10.7202/1055288ar d'utilisation que vous pouvez consulter en ligne.

https://apropos.erudit.org/fr/usagers/politique-dutilisation/ 
théoriques fondamentales des pensées hégélienne et technoscientifique pour en saisir en amont les divergences. L'approche de Lejeune, peut-être plus nuancée et moins catégorique à certains égards, semble au contraire refuser a priori toute divergence de fond, afin de souligner l'actualité de Hegel ${ }^{10}$. Mais une telle actualisation ne se fait pas toujours sans problème. Il ne s'agit évidemment pas ici de dire tout simplement que notre connaissance a tellement évolué depuis la mort de Hegel qu'il n'y aurait plus de dialogue possible. Il faut cependant reconnaître que la conception hégélienne de la technique, par exemple, n'est tout simplement pas transposable sans qu'il soit au préalable nécessaire d'apporter nuances et mises au point dans le contexte de ce qu'on pourrait appeler, si l'on me permet ce néologisme, $l^{\prime}$ ' autonomatisation ${ }^{11}$ " de la technique en technologie.

Malgré ces bémols concernant les conclusions générales de l'auteur qui apparaît parfois trop ambitieux dans sa volonté non seulement d'exposer l'anthropologie hégélienne et sa fonction au sein du système, mais d'actualiser en outre l'apport de Hegel aux enjeux contemporains soulevés par le transhumanisme et la technoscience, l'ouvrage reste des plus intéressants. On y trouve d'excellentes intuitions qui mériteraient de plus amples développements, mais aussi des présentations de qualité sur les conceptions hégéliennes de la folie, de la mort et de l'éducation. Cependant, si elles donnent matière à penser, les visées affichées par l'auteur dès l'introduction et les nombreuses pistes de réflexion qui jalonnent l'ouvrage laissent néanmoins une impression d'inachèvement et de manque de systématicité dans le traitement des enjeux soulevés.

EMMANUEL CHAPUT

Université de Montréal

\section{José L. Zalabardo, Representation and Reality in Wittgenstein's \\ Tractatus, Oxford, Oxford University Press, 2015, 263 pages}

Depuis trente ans, les études wittgensteiniennes tâchent de répondre à une question de méthode: comment convient-il de lire le Tractatus logicophilosophicus? Un tel trouble trouve sa source dans la célèbre pénultième remarque (6.54), par laquelle l'auteur explique que le comprendre c'est, en fin de compte, reconnaître ses remarques comme étant dépourvues de sens.

10. Pour marquer encore davantage l'opposition entre ces deux approches hégéliennes de la problématique technoscientifique que forment les ouvrages de Filion et Lejeune, notons que le premier se veut une critique des travaux du philosophe belge Gilbert Hottois, alors que c'est dans le prolongement des travaux de Hottois que s'inscrit Lejeune.

11. Conjonction de l'automatisation et de l'autonomisation de la technique qui justifierait le fait de parler de nos jours de technologie plutôt que de technique. 
À la fin des années I980, Cora Diamond lançait un défi ${ }^{1}$, celui de prendre cette remarque au sérieux et ainsi cesser de faire comme si Wittgenstein présentait des thèses philosophiques tout à fait pertinentes, quoiqu'ayant la fâcheuse particularité de ne pouvoir être formulées.

L'audace du livre de José Zalabardo est d'essayer de faire droit à cette contrainte de lecture, mais de telle sorte qu'elle n'interdise pas d'appréhender le Tractatus comme mettant en avant un ensemble cohérent de doctrines. Selon Zalabardo, reconnaître que les remarques de Wittgenstein sont dénuées de sens présuppose en effet de croire qu'elles «fournissent les seules réponses et solutions correctes aux questions et problèmes philosophiques qu'elles abordent ${ }^{2} »($ p. 3). Si ça n'était pas le cas, nous aurions affaire à de la mauvaise philosophie (à des questions pertinentes recevant des réponses dénuées de sens), et non pas à un rejet de l'entreprise philosophique (à des questions dont les seules réponses possibles s'avèrent être dénuées de sens).

Concentrant ses efforts sur l'une des questions centrales du Tractatus, celle de la représentation de la réalité par le langage, Zalabardo se propose de montrer comment les réponses qui y sont avancées peuvent sembler en constituer le dernier mot. C'est parce qu'une telle doctrine permet en apparence de répondre aux difficultés rencontrées par les théories du jugement de Russell entre I903 et I9I3 que Wittgenstein aurait d'abord été lui-même victime de l'illusion d'avoir produit l'unique solution aux problèmes concernant le rapport entre la réalité et sa représentation. Essentiellement invérifiable quoique convaincante, une telle hypothèse permet d'abord à l'auteur d'affirmer qu'en concevant la proposition comme un fait employé en tant qu'image d'un autre fait, Wittgenstein ne fait que modifier la théorie du jugement que Russell élaborait dans le manuscrit de La théorie de la connaissance ${ }^{3}$. À la suite de nombreuses versions de la théorie du jugement, que Zalabardo expose avec une grande clarté dans le chapitre I, Russell en vient à penser que juger que $\mathrm{A}$ aime $\mathrm{B}$, c'est produire en esprit un complexe qui parvient à représenter cette situation parce qu'il est lui-même composé à la fois des éléments du complexe représenté (A, B, aimer) et de sa forme, à savoir que «quelque chose est en relation avec quelque chose». C'est à cette seule condition qu'un jugement pourrait être à la fois faux (l'existence du complexe représentant ne suppose pas celle du complexe représenté) et déterminé (en incorporant la forme du complexe représenté, le complexe représentant détermine la manière dont les éléments y sont combinés). Une telle solution n'en est cependant pas une aux yeux de Wittgenstein, puisqu'elle suppose que la forme d'un complexe soit elle-même un fait, sans en avoir cependant la contingence. $\mathrm{La}$ vérité de « $\mathrm{A}$ aime $\mathrm{B}$ » dépendrait effectivement de celle de

1. Cora Diamond, "Throwing Away the Ladder", Philosophy, 63 (243), I988, p. 5-27.

2. Nous traduisons.

3. Bertrand Russell, Théorie de la connaissance. Le manuscrit de 1913, Paris, Vrin, 2002. 
"quelque chose est en relation avec quelque chose», proposition que Russell estime à tort être purement formelle et par conséquent nécessairement vraie.

Le chapitre II est alors en mesure de mettre au jour aussi bien l'originalité de la théorie de l'image du Tractatus que sa continuité avec la théorie du jugement comme relation multiple de Russell. Le legs de cette dernière réside dans l'idée que c'est en faisant usage d'un fait, c'est-à-dire d'une certaine combinaison de choses, que l'on peut en représenter un autre, correctement ou incorrectement. Mais là où, pour ce faire, Russell identifie les constituants du complexe représentant à ceux du complexe représenté et y adjoint la présence de la forme du complexe dans le jugement, Wittgenstein, lui, rompt avec cette conception chosiste de la forme en procédant de manière inverse. L'identité de l'image et de ce qu'elle dépeint ne réside pas dans leurs constituants (par convention, tel élément de l'image tient lieu de tel objet du monde), mais dans la manière par laquelle ils s'agencent. Ainsi, le fait que le chat soit sur la chaise peut représenter le fait que le vase est sur la table, à condition de décider que le chat tienne lieu du vase et que la chaise tienne lieu de la table. La représentation de la réalité s'explique donc par la possibilité que les choses représentées soient dans le même rapport que le sont les éléments de l'image. À la fin du chapitre II, Zalabardo a ainsi montré que la solution tractatuséenne au problème de la possibilité du faux demeure russelienne et que l'apport de Wittgenstein est de rendre impossible une représentation illogique. Là où la théorie du jugement de Russell ne permet pas d'exclure des jugements portant sur des combinaisons absurdes, telles que «le chat chaise la table», Wittgenstein montre qu'ils ne sauraient pas même être formés, toute image étant un usage d'une certaine combinaison actuelle d'objets. S'il est possible de former une image, ce qu'elle dépeint doit également être possible.

Une telle généalogie de la théorie de l'image s'expose à une objection évidente: tandis que Russell pense la représentation comme jugement, et ainsi comme relation entre le sujet et le monde, c'est par son absence que le sujet s'illustre dans le Tractatus. Zalabardo s'en explique au chapitre III: en ne faisant pas intervenir le sujet de la représentation, Wittgenstein adopterait une "approche disciplinée» (p. I07), car fidèle à la leçon tractuséenne selon laquelle il est impossible de représenter le sujet représentant le monde. Une phrase telle que « $\mathrm{A}$ croit que $\mathrm{B}$ aime $\mathrm{C}$ » conduit en effet à une alternative insatisfaisante: ou bien ce qu'elle représente est une relation dont les deux relata sont le sujet $\mathrm{A}$ et l'unité « $\mathrm{B}$ aime $\mathrm{C} »-$ mais on ne comprend pas à quoi peut être lié le sujet si sa croyance est fausse - , ou bien elle représente une relation entre le sujet $\mathrm{A}$ et les éléments $\mathrm{B}, \mathrm{C}$ et «aime » ainsi unifiés par la croyance -, mais on supposera alors à tort que le verbe "aimer" n'est pas l'expression d'une relation. Face à ce paradoxe, Russell concluait à une "énigme de la nature de la croyance ${ }^{4}$ ». Il reviendrait à

4. Bertrand Russell, Écrits de logique philosophique, Paris, PUF, I989, p. 384. 
Wittgenstein de lui avoir signalé cette difficulté et d'avoir su en apprécier la radicalité en admettant que ce qui ne peut être représenté n'existe pas. À l'inverse, Zalabardo s'autorise d'une "approche indisciplinée » pour expliciter la théorie de l'image en ne s'interdisant pas d'y faire intervenir le sujet. La pertinence d'une telle distinction des approches «disciplinée » et "indiciplinée » ne va néanmoins pas de soi: si Wittgenstein s'était véritablement interdit de parler de ce dont il dit que l'on ne peut parler, le Tractatus serait un ouvrage plus court encore qu'il ne l'est déjà. Quelle que soit cependant la valeur de cette distinction, Zalabardo parvient à montrer dans les chapitres I à III que l'identité formelle entre le fait qui représente et le fait représenté permet d'expliquer que toute représentation intègre nécessairement le mode de combinaison des objets de l'état de choses représenté.

Wittgenstein apporterait ainsi une solution au problème du mode de combinaison, mais adopterait une tout autre attitude envers le problème de l'unité. Il n'y aurait pas à expliquer comment un fait, notamment propositionnel, est produit à partir de ses éléments, puisque le fait est lui-même l'unité minimale et indivisible de la réalité et de sa représentation. Les chapitres IV et V s'attachent à expliciter cette position en en soulignant d'abord l'origine frégéenne. De même que, selon Frege, les concepts et leurs expressions ne précèdent pas les jugements mais sont mis au jour par l'analyse de ces derniers, la proposition précède, selon Wittgenstein, les expressions qu'elle contient. S'il n'est pas inédit d'identifier une généralisation à toutes les expressions de cette thèse frégéenne dans le Tractatus et ainsi de rejeter l'idée traditionnelle d'un atomisme logique qui serait commun à Russell et à Wittgenstein, Zalabardo s'efforce d'en exposer le détail et les conséquences. Il propose de comprendre les constituants propositionnels comme autant de traits caractéristiques communs à un ensemble de propositions dont ils sont abstraits. C'est cela même qui permet d'expliquer que les constituants propositionnels contiennent en eux-mêmes leurs propres possibilités de combinaison: abstraits à partir de faits, ils spécifient quelles sont les combinaisons possibles en mettant en jeu la manière par laquelle ils sont combinés les uns aux autres. L'inspiration est une fois encore frégéenne, le caractère insaturé des concepts indiquant chez ce dernier qu'ils contiennent en eux-même la structure des phrases dans lesquelles leurs expressions sont susceptibles d'apparaître.

Concevoir les objets et les noms comme des traits communs des états de choses et des propositions permettrait également, selon Zalabardo, de comprendre en quel sens Wittgenstein affirme qu'ils sont simples. En tant que trait commun aux propositions «Dimitri aime Florian» et «Dimitri est plus petit que Valère », le constituant propositionnel "Dimitri entretenant une relation binaire avec un individu» n'est pas composé et ne saurait être lui-même divisé. Pourtant, un passage notoirement difficile du Tractatus $(2.02-2.02 \mathrm{I} 2)$ soutient que la raison pour laquelle les objets sont simples est qu'ils constituent la substance du monde et que si le monde n'avait pas 
de substance, il serait impossible de le représenter, dans la mesure où le sens d'une proposition dépendrait de la vérité d'une autre. Selon la plupart des commentateurs, que les objets soient simples signifie qu'il s'agit d'entités isolées dont l'existence est nécessaire: s'il s'agissait de complexes, ils pourraient ne pas exister et le sens d'une proposition $p$ dépendrait alors de la vérité d'une proposition $q$ affirmant l'existence des objets sur lesquels porte p. Zalabardo rejette radicalement cette lecture (à laquelle il consacre l'un des deux appendices de son ouvrage) et propose de comprendre tout autrement la notion de "substance». Celle-ci ne renverrait pas au mode d'existence des objets, mais au fait qu'ils incarnent la forme, c'est-à-dire la possibilité de leurs combinaisons. Si le monde n'avait pas de substance, si les objets ne contenaient pas en eux-mêmes la possibilité de leurs combinaisons, le sens de $p$ dépendrait de la vérité de $q$, qui affirmerait la possibilité de la combinaison dépeinte par $p$. C'est là une interprétation remarquable qui a le mérite de vider la notion de "substance" de son caractère métaphysique, mais dont on aimerait qu'elle précise pourquoi Wittgenstein recourt tout de même à ce concept traditionnel.

Comme l'expose le chapitre V, c'est également en concevant les constituants propositionnels comme traits caractéristiques à un ensemble de propositions que l'on peut rendre raison des limites de la représentation. Les propositions dans lesquelles un prédicat est attribué à lui-même, celles qui portent sur elles-mêmes ou encore celles qui représentent leur propre exemplification sont toutes impossibles. Non pas qu'il faille les exclure à la suite d'un examen de la signification de leurs signes comme le propose par exemple la théorie des types de Russell, mais parce que, de manière purement syntaxique, il est impossible de les construire: elles contrediraient les possibilités de combinaison inhérentes aux constituants propositionnels.

Dans les cinq premiers chapitres, Zalabardo choisit de considérer la théorie de l'image comme si elle s'appliquait à la façon par laquelle les propositions que l'on emploie usuellement représentent la réalité. Mais l'on sait pourtant que le Tractatus déclare que toute proposition usuelle, comme «le chat est sur la table», est en réalité une fonction de vérité de propositions élémentaires, propositions les plus fondamentales dont Wittgenstein affirme la possibilité sans pouvoir cependant en donner un seul exemple. Le sixième et dernier chapitre, qui est le plus critique, évalue une telle doctrine tout en en mettant au jour les motifs et les limites. Les propositions élémentaires ont deux particularités, qui ont placé bien des lecteurs dans l'embarras: elles sont logiquement indépendantes les unes des autres, et leurs constituants ne tiennent lieu que d'objets simples. Zalabardo montre magistralement que ce sont là deux exigences de ce qu'il appelle le "formalisme épistémologique " de Wittgenstein (p. I9I), qui soutient que nous parvenons à connaître les rapports logiques entre les propositions par le seul examen de leurs structures. Si les valeurs de vérité des propositions élémentaires n'étaient pas mutuellement indépendantes, ou si leurs constituants tenaient lieu de com- 


\section{Philosophiques / Automne 2018}

plexes, déterminer qu'une proposition est une conséquence logique d'autres propositions supposerait de devoir consulter de surcroît les états de choses qu'elles représentent, et non pas simplement leurs propositions élémentaires. L'auteur estime que cette position présente un intérêt certain pour l'épistémologie de la logique, mais en souligne les limites: en distinguant les propositions élémentaires et les propositions usuelles, Wittgenstein échouerait à prouver que sa théorie de l'image s'applique également à ces dernières.

La conclusion s'attache à montrer que si les doctrines examinées ont toutes une certaine valeur philosophique, elles rencontrent néanmoins des difficultés insurmontables. De telle sorte que, selon Zalabardo, Wittgenstein n'ayant pu prouver que ses solutions sont les seules possibles - certaines n'étant pas du tout des solutions -, il ne peut davantage nous conduire à admettre que son propos n'est que non-sens. En supposant que telle était l'ambition du Tractatus, Zalabardo offre une lecture approfondie et rigoureuse du contenu même de l'ouvrage. Il s'agit à ce titre d'un livre d'une grande importance, dont on peut supposer qu'il inaugurera une nouvelle manière de lire le Tractatus dans les études wittgensteiniennes. Il est seulement regrettable que celle-ci ne permette pas d'honorer la demande formulée en 6.54 .

JONATHAN GOMBIN

Université Bordeaux Montaigne 